journal homepage: http://chimie-biologie.ubm.ro/carpathian_journal/index.html

\title{
HPLC QUANTIFICATION OF THE CHEMICAL CONSTITUENTS FROM INDIGENOUS FRUITS AND VEGETABLES OF INDIAN HIMALAYAN REGION
}

\author{
Tanveer Alam ${ }^{1}$, Murtaza Gani ${ }^{1 凶}$, Rukhsana Rahman $^{2}$, Khalid ul Islam Rather ${ }^{3}$ \\ ${ }^{1}$ Department of Chemistry, KLDAV PG College Roorkee Uttrakhand, Affiliated to Department of Chemistry, \\ HNB Garhwal University Srinagar (Garhwal) Uttrakhand India. \\ ${ }^{2}$ Division of Food Science and Technology, Shere Kashmir University of Agricultural Sciences \& \\ Technology, Jammu, India. \\ ${ }^{3}$ High End Instrumentation Lab, Public Health Laboratory Dalgate Srinagar J \& K India. \\ 凶kmurtazakmg@gmail.com \& tanvdav@gmail.com \\ https://doi.org/10.34302/crpjfst/2021.13.3.7 \\ Article history: \\ Received: \\ 15 May 2021 \\ Accepted: \\ 2 August 2021 \\ Keywords: \\ Analysis; \\ Crops; Gradient; \\ HPLC; \\ Minor; \\ Phytochemicals.

\begin{abstract}
The purpose of the present work was to determine the phytochemical profiles by HPLC of the indigenous fruits and vegetables. The phenolic contents showed diverse variation in the selected fruits and vegetables. Development of genuine and dependable analytical methods with profile marker phytoconstituents in an extract containing a mixture of several components is a challenging task. A simple, rapid, precise and reliable HPLC method was developed for the quantification of phytochemicals from the extracts of selected minor fruits and vegetables. The Taraxacum officinale genus comprised a mixture of different bioactive compounds belonging to different chemical types, such as flavonoids, sesquiterpenes, triterpenes, phenolic acids, sterols. Malva neglecta contains different compounds including several phenolic acids, flavonoids and some non-phenolic compounds. Caffeoylquinic acids (3-, 4-, and 5-O-caffeoylquinic acids and 3,5dicaffeoylquinic acid) are mainly present in Cydonia oblonga pulps. Three different hydroxycinnamic acid derivatives (neochlorogenic acid, p-coumaroylquinic acid and chlorogenic acid) were detected and quantified in Prunus avium.
\end{abstract}

\section{Introduction}

Indigenous fruits and vegetables are widely used by various traditions and cultures to fulfil energy source. They are potent sources of phytochemical components like polyphenols, antioxidants, minerals, vitamins, etc. Due to the special agro-climatic conditions in the temperate Himalayas of India, Kashmir has a variety of fruits and vegetables. The indigenous crops of the area are used by people to meet their basic needs. The indigenous fruits selected were Cherry (Prunus avium) and Quince (Cydonia oblonga) and the selected vegetables were Handh (Taraxacum officinale) \& Sustchal (Malva neglecta).

Handh has potential health benefits due to the presence of phenolics, flavonoids, coumarins, terpenoids, sesquiterpene lactones, carotenoids, chlorophylls, dietary fibre and alkaloids (Colle et al., 2012; GonzalezCastejon, Visioli, \& Rodriguez-Casado, 2012). Among flavonoids, apigenin-7-Oglycoside, luteolin-7-O-glycoside and naringenin-7-Oglycoside were identified and furthered analysed for their antimicrobial and antioxidant activities (Kenny et al., 2015).

Malva neglecta is referred to as Khebaiz or Khobb eiza in Arabic and belongs to Malvaceae family and is wildly grown in the Northern Border Province, Saudi Arabia. It has been traditionally used for insect bites, bladder infection, burns, inflammation, ulcers and wounds, as astringent, demulcent, diuretic, expectorant and laxative. Some of the 
phytoconstituents reported in the literature are Quinic acid, Aconitic acid, Chlorogenic acid, Caffeic acid, Coumaric acid, Rutin, Hyperoside, Myricetin, Fisetin, Coumarin, Quercetin, Naringenin, Luteolin, Kaempferol, Apigenin, Rhamnetin and Chysin (Nesrin et al., 2017; Guder et al., 2012; Karak 2019; Essafi Benkhadir et al., 2012; Fattouch et al., 2007).

A large number of polyphenolic constituents were identified in quince fruit; in particular, flavan-3-ols such as Epicatechin, Procyanidin B2, eight Hydroxycinnamates, derivatives of Caffeoylquinic acid (CQA) and Coumaroylquinic acid, Kaempferol and Quercetin derivatives (Nagahora et al., 2013; Wojdylo et al., 2013; Hamauzu et al., 2005). The analysis of the phenolic profile indicated that derivatives of Quercetin and Kaempferol were minor components of quince fruit, while Procyanidins and Chlorogenic acid (CGA) derivatives constituted the majority of the polyphenolic fraction (Bystricka et al., 2017; Crozier et al., 2009; Strek et al., 2007; Joana et al., 2019). The other main component of quince are proanthocyanidins, compounds widely known for their antioxidant and protective effects on cardiovascular and cancer-related diseases, in part due to their ability to modulate pro-inflammatory and carcinogenic signals. (Joana et al., 2019; Serra et al., 2011). Procyanidins from quince fruit have been studied for their chemoprevention activity, and preliminary investigation indicated an effect against enzymes and receptors, suggesting a role in cancer prevention (Serra et al., 2011).

Sweet cherries are an excellent source of phytochemicals, namely phenolic compounds (Bastos et al., 2015). The concentration of polyphenols can vary between different sweet cherry cultivars and in different parts thereof (Tural and Koca 2008). Studies have shown that the antioxidant capacity in the stem is greater than the antioxidant capacity in the pulp, leading people to believe that this is due to the higher concentration of phenols found here as well. (Wang et al., 2017).The levels of phenolic compounds can be affected by several factors, and this is associated to both climatic and agronomic conditions (Toydemir et al., 2013; Liu 2013). Another study in acid cherry (Prunus cerasus L.) also allowed the phenolic composition to be determined using a high performance liquid chromatography coupled with fluorescence detection (HPLCFLD) (Monteiro Egydio et al., 2013). A study of several sweet cherry cultivars using highperformance liquid chromatography equipment coupled to ultraviolet (HPLC-UV) and diode array (HPLC-DAD) (Toydemir et al., 2013) is also reported.

Currently, some studies support the idea that a healthy diet can prevent the development of certain diseases. (Liu 2013). In addition, research works have shown the high relationship between the consumption of fruits in prevention of various chronic health problems (Monteiro Egydio et al., 2013). Not only fruit pulp, but also peels and seeds from dietary plants and traditional medicinal herbs, play an important role in health because of their nutritional, antioxidant properties and wealth due to bioactive compounds. It is also interesting to know the presence and concentration of polar compounds (such as organic acids), because these compounds affect sensory and sensory properties. In addition, organic acids can be used as a chemical indicator of maturity, bacterial activity or storage conditions, because these compounds have good stability during processing and storage. (Sandin-Espana et al., 2016; Vagelas and Sugar, 2020).

Currently, there are few studies on phenolic compounds and organic acids of the selected minor fruits and vegetables. Due to the few available research studies about the characterization of bioactive compounds in selected minor fruits and vegetables, the aim of the present work was to comprehensive and tentative identify phenolic compounds and organic acids in the edible part of selected minor fruits and vegetables. This study presents the selected minor fruits and vegetables as potential sources of bioactive compounds with applications in the food, pharmaceutical and cosmetic industries.

\section{Materials and Methods}

\subsection{Chemicals and Reagents}

HPLC grade formic acid, Methanol, Water \& Acetonitrile were purchased from Himedia (LBS, Marg, Mumbai, India). 
Solvents were filtered using a Solvent Filtration Apparatus 58,061 (Supelco, Bellefonte, PA, USA). Double- deionised water was obtained with a Milli-Q-system from Millipore (Bangalore India). The standard compounds Quinic acid, Malic acid, tr-Aconitic acid, Gallic acid, Chlorogenic acid, Protocatechuic acid, Tannic acid, tr-Caffeic acid, Vanillin, P- Coumaric acid, Rosmarinic acid, Rutin, Hesperidin, Hyperoside, 4-OHBenzoic acid, Salicylic acid, Myricetin, Fisetin, Coumarin, Quercetin, Naringenin, Hesperidin, Luteolin, Kaempferol, Apigenin, Rhamnetin, Chyrsin, 3-O-Caffeoyl quinic acid, 4-O-Caffeoyl quinic acid, 5-O-Caffeoyl quinic acid, 3,5-di Caffeoyl quinic acid, Quercetin 3- galactoside, Kaempferol glycoside, Kaempferol 3-glycoside, Kaempferol 3-Rutinoside, NeoChlorogenic acid, P- Coumaryl quinic acid, Cyanidin 3glucoside, Cyanidin 3- Rutinoside, Peonidin 3glucoside, Pelargonidin 3- Rutinoside, Peonidin 3- Rutinoside, Catechin, Epicatechin, 9- Cis Violaxanthin, Neochrome, All- transNeoxanthin, All-trans-Violaxanthin, 9-cisNeoxanthin, Luteoxanthin, cis-Violaxanthin, Antheraxanthin, 9-cis-Violaxanthin, 13- cisLutein, All trans Lutein, All trans Zeaxanthin, 9 cis- Lutein, were purchased from Sigma Aldrich (St. Louis, MO, USA).

\subsection{Extraction}

A solid- liquid extraction was used to extract the polar fraction. Briefly, $0.5 \mathrm{~g}$ of freeze-dried sample powder was dissolved in $15 \mathrm{~mL}$ of a solution of methanol/ water (80: 20 $\mathrm{v} / \mathrm{v})$. The mixture was placed in a water bath for $15 \mathrm{~min}$ at room temperature and then it was centrifuged for $15 \mathrm{~min}$ at $5000 \mathrm{rpm}$, the supernatant was removed, and extraction was repeated once more. The supernatants were collected, evaporated and reconstituted in 2 $\mathrm{mL}$ of methanol/ water $(80: 20, \mathrm{v} / \mathrm{v})$. The final extracts were filtered with regenerated cellulose filters $0.2 \mu \mathrm{m}$, (Millipore, Bedford, MA, USA) and stored at $-18{ }^{\circ} \mathrm{C}$ until the analyses.

\subsection{UPLC DAD Analyses}

The extracts obtained by solid-liquid extraction were analysed by using UPLC coupled to DAD in order to identify phenolic compounds. An Agilent 1290 Infinity II- LC system (Agilent Technologies, Waldbronn Germany) equipped with a vacuum degasser, autosampler, quaternary pump, and DAD was used for the chromatographic determination. All the methods were validated and optimized.

For Taraxacum officinale, the separation was done using an Agilent Poroshell Eclipse plus C30 column $(4.6 \times 250 \mathrm{~mm}, 5 \mu \mathrm{m})$ from Agilent Technologies operating at a flow rate of $1 \mathrm{~mL} / \mathrm{min}$ and $40^{\circ} \mathrm{C}$, thorough out the gradient. The mobile phases used were water with $\mathrm{MeOH} / \mathrm{ACN}$ in the ratio of $79: 14: 7$ (Phase A) and Methylene chloride (Phase B), and the solvent gradient changed according to the following conditions: $95 \% \mathrm{~A}$ in the beginning and maintained for $9 \mathrm{~min}$, decreased to $85 \% \mathrm{~A}$ in $23 \mathrm{~min}, 83 \% \mathrm{~A}$ in $33 \mathrm{~min}, 71 \% \mathrm{~A}$ in $35 \mathrm{~min}, 70 \% \mathrm{~A}$ in $45 \mathrm{~min}, 66 \% \mathrm{~A}$ in $66 \mathrm{~min}$ and returned to original ratio in $71 \mathrm{~min}$. The injection volume was $20 \mu \mathrm{L}$ and chromatograms were recorded from 190-600 $\mathrm{nm}$ with $450 \mathrm{~nm}$ as maximum wavelength.

For Malva neglecta, the chromatographic separation was performed on a $\mathrm{C} 18$ reversedphase Inertsil ODS-4 $(150 \mathrm{~mm} \times 4.6 \mathrm{~mm}$, $3 \mu \mathrm{m})$ analytical column. The column temperature was fixed at $40^{\circ} \mathrm{C}$. The elution gradient consisted of mobile phase A (water, $5 \mathrm{mM}$ ammonium formate and $0.1 \%$ formic acid) and mobile phase $\mathrm{B}$ (methanol, $5 \mathrm{mM}$ ammonium formate and $0.1 \%$ formic acid). Use the gradient program $\mathrm{t}(\mathrm{min})$ of solvent $\mathrm{B}$ in the following proportions, $\%$ B: (0.40), (20.90), (23.99.90), (24.40), (29.40). The solvent flow rate was maintained at $0.5 \mathrm{ml} /$ min and the injection volume was stable at 4 $\mu 1$.

For Cydonia oblonga, chromatographic separation was carried out on a LiChroCART column $(250 \times 4 \mathrm{~mm}, \mathrm{RP}-18,5-\mu \mathrm{m}$ particle size, Merck, Darmstadt, Germany) using two solvents: water/formic acid (19:1) (A) and methanol (B); starting with 5\% methanol and installing a gradient to obtain $15 \%$ B at $3 \mathrm{~min}$, $25 \% \mathrm{~B}$ at $13 \mathrm{~min}, 30 \% \mathrm{~B}$ at $25 \mathrm{~min}, 35 \% \mathrm{~B}$ at $35 \mathrm{~min}, 45 \% \mathrm{~B}$ at $39 \mathrm{~min}, 45 \% \mathrm{~B}$ at $42 \mathrm{~min}$, $50 \% \mathrm{~B}$ at $44 \mathrm{~min}, 55 \% \mathrm{~B}$ at $47 \mathrm{~min}, 70 \% \mathrm{~B}$ at $50 \mathrm{~min}, 75 \% \mathrm{~B}$ at $56 \mathrm{~min}$, and $80 \% \mathrm{~B}$ at 60 min. The flow rate was $0.9 \mathrm{~mL} / \mathrm{min}$, and the injection volume was $80 \mu \mathrm{L}$. Detection was carried out at 270, 320, and $350 \mathrm{~nm}$. 
In case of Prunus avium, the HPLC equipment was used with a diode array detector (DAD). The system consists of a binary pump, a degasser and an autosampler. The chromatographic column used is Beckman Ultrasphere ODS (Roissy CDG, France): $4.6 \mathrm{~mm} \times 250 \mathrm{~mm}, 5 \mu \mathrm{m}$, equipped with a guard column of $4.6 \mathrm{~mm} \times 10 \mathrm{~mm}$. The mobile phase consists of two solvents: solvent A, water/formic acid $(95: 5 ; \mathrm{v} / \mathrm{v})$ and solvent $\mathrm{B}$, acetonitrile/solvent A $(60: 40 ; \mathrm{v} / \mathrm{v})$. Phenolic compounds were eluted under the following conditions: $1 \mathrm{~mL} / \mathrm{min}$ flow rate and the temperature was set at $25{ }^{\circ} \mathrm{C}$, isocratic conditions from 0 to $10 \mathrm{~min}$ with $0 \% \mathrm{~B}$, gradient conditions from $0 \%$ to $5 \% \mathrm{~B}$ in 30 min, from $5 \%$ to $15 \%$ B in 18 min, from $15 \%$ to $25 \% \mathrm{~B}$ in $14 \mathrm{~min}$, from $25 \%$ to $50 \% \mathrm{~B}$ in 31 min, from $50 \%$ to $100 \% \mathrm{~B}$ in $3 \mathrm{~min}$, followed by washing and reconditioning the column.

\section{Results and discussions}

The separation of all the compounds in complex extract is one of the challenging tasks in analytical HPLC.

\subsection{Taraxacum Officinale (Handh)}

According to current knowledge, the genus Taraxacum includes a mixture of different biologically active compounds belonging to different chemical types, such as flavonoids, sesquiterpenes, triterpenes, phenolic acids, sterols, etc. Among them, flavonoids and phenolic acids were the more abundant metabolites, which always had similar structure skeleton. It has been well established that a C30 column can provide a much better resolution than a $\mathrm{C} 18$ column in separating carotenoids and their geometrical isomers (Chen et al., 2004; Dugo et al., 2008). Initially a gradient solvent system of methanol/acetonitrile/water $(84: 14: 2, \mathrm{v} / \mathrm{v} / \mathrm{v})$ and methylene chloride developed by Inbaraj et al., (2008) was used to separate the various carotenoids in $\mathrm{T}$. formosanum by a YMC C30 column $(250 \mathrm{~mm} \times 4.6 \mathrm{~mm}$ I.D., particle size $5 \mu \mathrm{m})$. However, several carotenoid peaks overlapped, which may be due to the high concentration of solvent in the mobile phase. Therefore, it is necessary to reduce the strength of the solvent by increasing the polarity of the mobile phase. After various studies, a gradient mobile phase of methanol/acetonitrile/water $(79: 14: 7, \mathrm{v} / \mathrm{v} / \mathrm{v})(\mathrm{A})$ and methylene chloride (B) was developed to separate 25 carotenoids and their geometrical isomers within $66 \mathrm{~min}$ (Fig. 1), with the retention times ranging from 11.54 to $64.22 \mathrm{~min}$, retention factor from 2.51 to 18.57 , separation factor from 1.01 to 1.44 and peak purity from 88.3 to $99.8 \%$ (Table 1). Most carotenoid peaks were adequately resolved (Fig. 1), implying both solvent strength and selectivity of mobile phase to sample components were properly controlled.

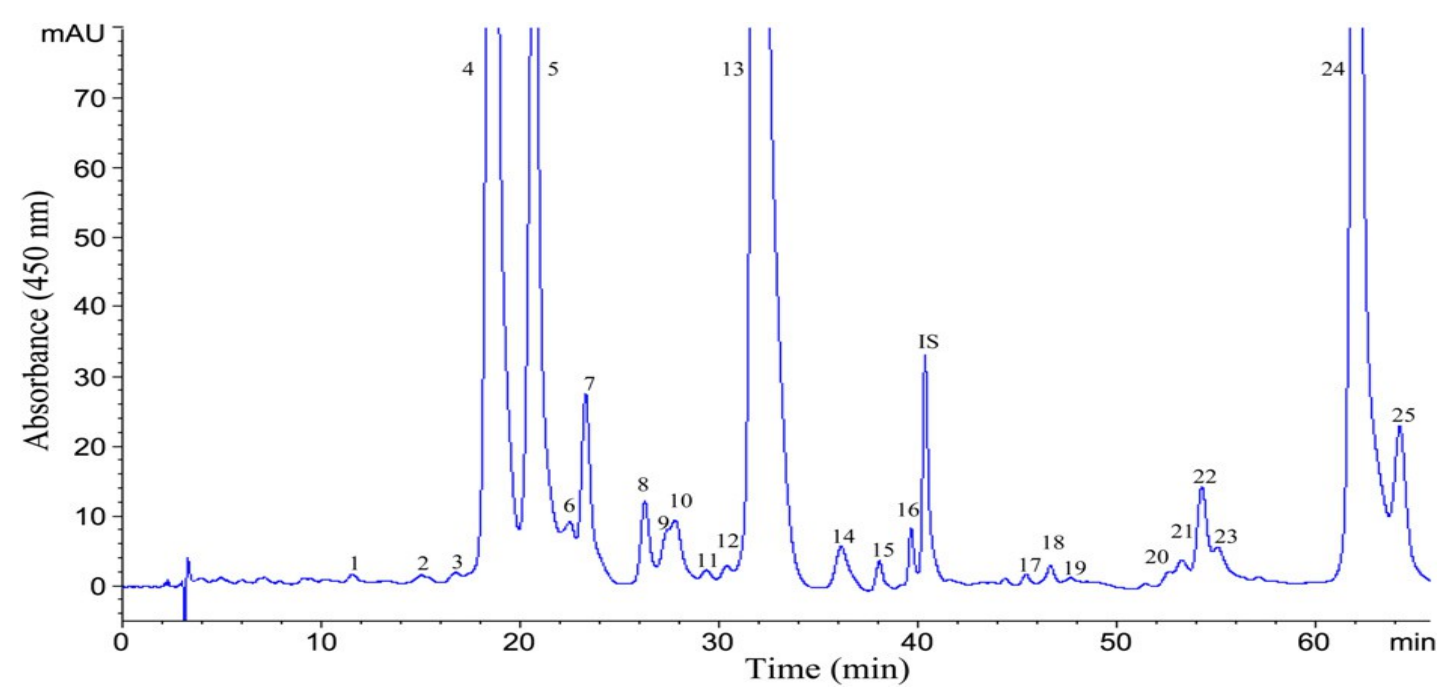

Figure 1. HPLC chromatogram of Standards of T. officinale (column: C30, mobile phase: (A) methanol-acetonitrile-water $(79: 14: 7, \mathrm{v} / \mathrm{v} / \mathrm{v}),(B)$ methylene chloride, flow rate: $1 \mathrm{~mL} / \mathrm{min}$, detection wavelength: $450 \mathrm{~nm}$ ). 
In Fig. 1, peak 1 was identified as 9- or 9'cis- violaxanthin. Peak 2 was identified as neochrome. Both peaks of 3 and 4 were identified as all -trans forms of neoxanthin and violaxanthin, respectively. Peak 5 was identified as 9 or 9'- cis-neoxanthin. Both peaks 6 and 10 were identified as luteoxanthin based on absorption spectra and mass spectra characteristic and compared with that reported by Chen et al., (2004) and Dugo et al., (2008). Following the same approach, peaks 7 and 9 were identified as cis-violaxanthin and 9 or 9'- cis violaxanthin, respectively (MelendezMartinez et al., 2008; Chen et al., 2004). Peak 8 was identified as antheraxanthin. Peaks 13, 14,17 and 24 were positively identified as alltrans forms of lutein, zeaxanthin, $\beta$ cryptoxanthin and $\beta$-carotene, respectively. In several similar studies the major carotenoids, including neoxanthin, violaxanthin, $\beta$ carotene, zeaxanthin, $\beta$ - cryptoxanthin and antherxanthin were also detected in Taraxacum officinale.

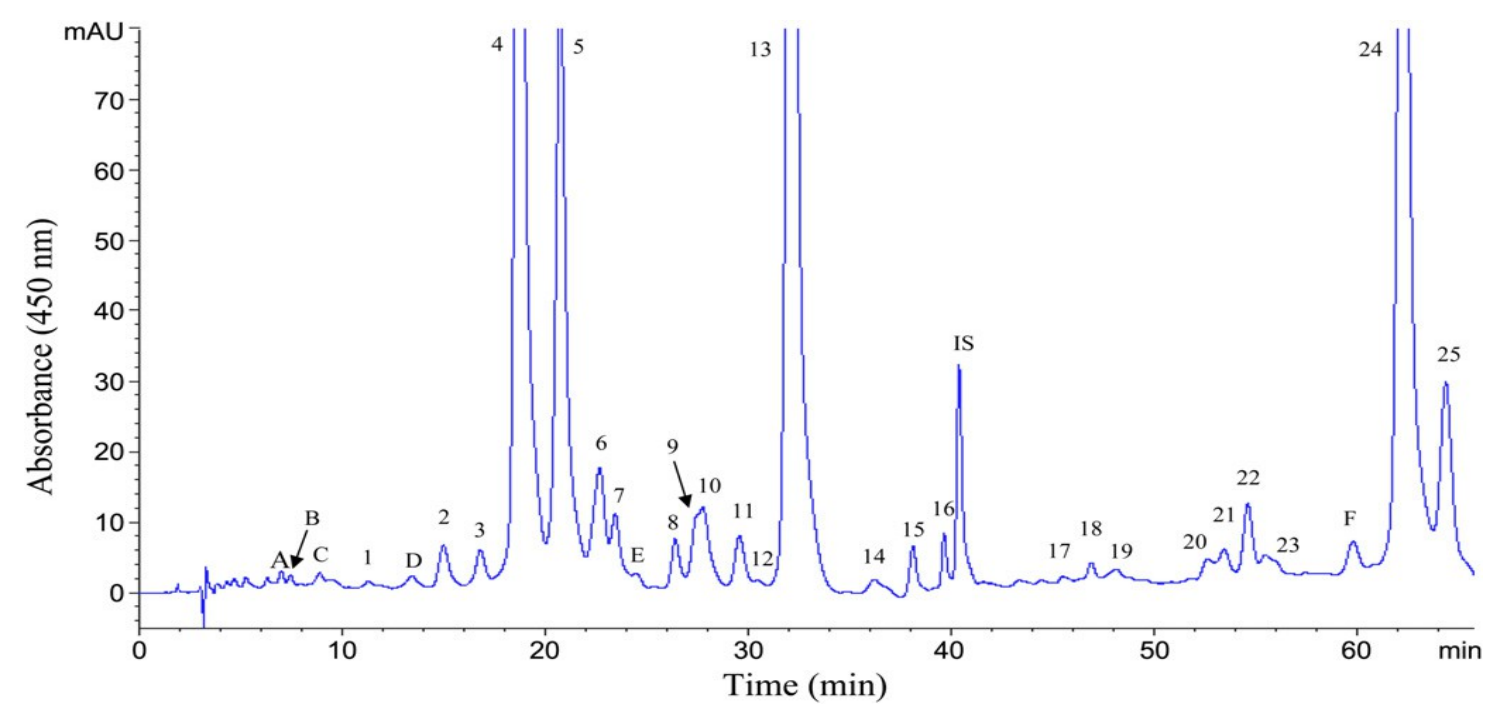

Figure 2. HPLC chromatogram of extract of T. officinale. Peak numbers with alphabetical letters (A-F) indicate additional compounds identified in extract fraction isolated by column chromatography, while 1-25 denote the same standard compound.

Fig. 2 shows the HPLC chromatogram of various carotenoids in carotenoid fraction isolated from Taraxacum extract. Following the same identification and quantitation criteria, a total of 31 carotenoids were separated and identified (Faria et al., 2009; Zepka et al., 2009). The peaks marked as A-F were identified as Auroxanthin, 13- cisneoxanthin, Violaxanthin and 9-cis- $\beta$ carotene.

\subsection{Malva Neglecta (Sustchal)}

In the quantitative analysis of phenolic compounds various studies exist in literature reporting the use of HPLC (Mousavi et al., 2015; Fahimi et al., 2016). Figure 3 depicts the chromatogram of the standards of Malva neglecta.

Therefore, an accurate quantitative method was developed on HPLC for the analyses of twenty-seven compounds. The methanol extracts of $M$. neglecta were screened to quantify their phenolic composition by using an Agilent 1290 Infinity II- LC system.

This accurate method allowed us to qualify and quantify 27 different compounds including several phenolic acids, flavonoids and some non-phenolic compounds. To summarize, this is the first study to screen 27 compounds in $\mathrm{M}$. neglecta. It is rich in terms of phenolic acids.

The detected compounds were Quinic acid, Malic acid, tr- Aconitic acid, Gallic acid, Chlorogenic acid, Protocatechuic acid, Tannic acid, tr- caffeic acid, Vanillin, p- Coumaric acid, Rosmarinic acid, Rutin, Hesperidin, Hyperoside, 4- OH Benzoic acid, Salicylic acid, Myricetin, Fisetin, Coumarin, Quercetin, Naringenin, Hesperedin, Luteolin, Kampferol, Apigenin, Rhamnetin and Chysin (Fig. 4). 


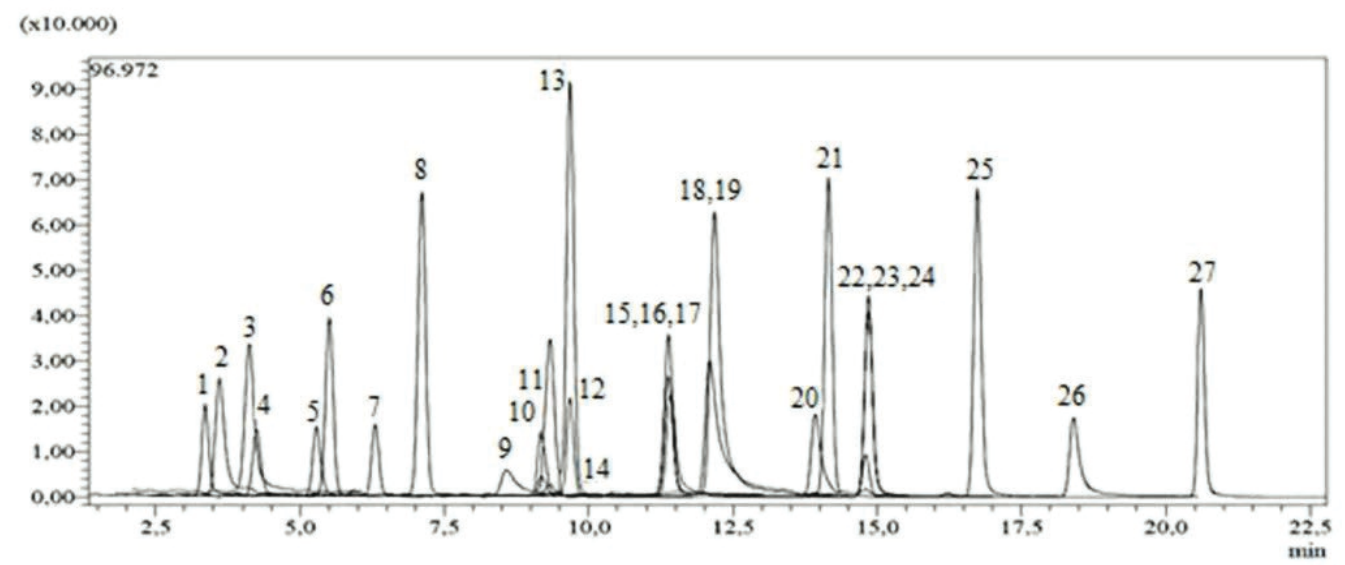

Figure 3. HPLC chromatogram of Standards of Malva neglecta.

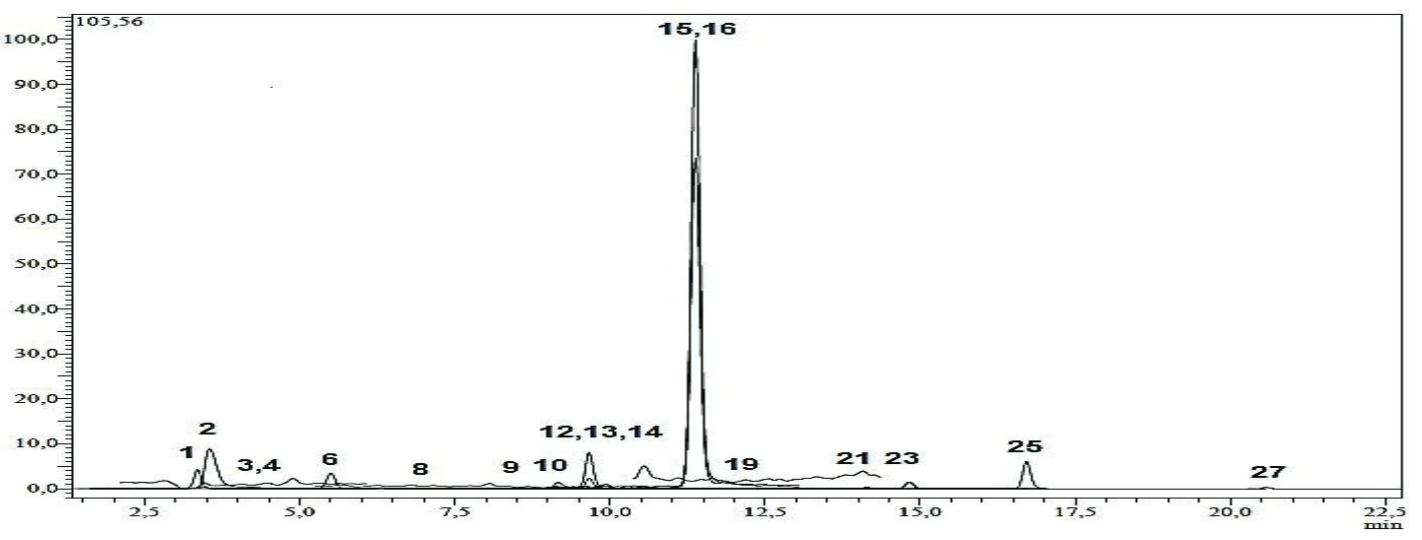

Figure 4. HPLC profile of Malva Neglecta methanol Extract. 1: Quinic acid, 2: Malic acid, 3: tr-Aconitic acid, 4: Gallic acid, 5: Chlorogenic acid, 6: Protocatechuic acid, 7: Tannic acid, 8: tr- caffeic acid, 9: Vanillin, 10: p-Coumaric acid, 11: Rosmarinic acid, 12: Rutin, 13: Hesperidin, 14: Hyperoside, 15: 4-OH Benzoic acid, 16: Salicylic acid, 17: Myricetin, 18: Fisetin, 19: Coumarin, 20: Quercetin, 21: Naringenin, 22: Hesperetin,

23: Luteolin, 24: Kaempferol, 25: Apigenin, 26: Rhamnetin, 27: Chrysin.

\subsection{Cydonia oblonga (Quince)}

All quince pulps presented the same chemical profile, composed by at least five identified phenolic compounds: 3-, 4-, and 5O-caffeoylquinic acids, 3, 5-dicaffeoylquinic acid, and rutin. Fig. 5 depicts the chromatogram of the standards of Cydonia oblonga.

The first group (39-46 min) was formed by five major peaks (peaks 5 to 9) and the second group (51-56 min) was formed by three peaks (Fig. 6).

All of the peaks showed identical spectral profile, with two maxima at 257 and 353-355 $\mathrm{nm}$, which indicated that they could be flavonols or flavonol derivatives. HPLC analyses provided interesting information on the two mentioned groups of flavonoids. Quince fruit composition was characterised by the presence of 4- caffeoylshikimic acid, 4caffeoyl quinic acid, quercetin-3, 7diglucoside, kaempferol-3-O- rhamnoside and kaempferol-7-O-glucoside. 


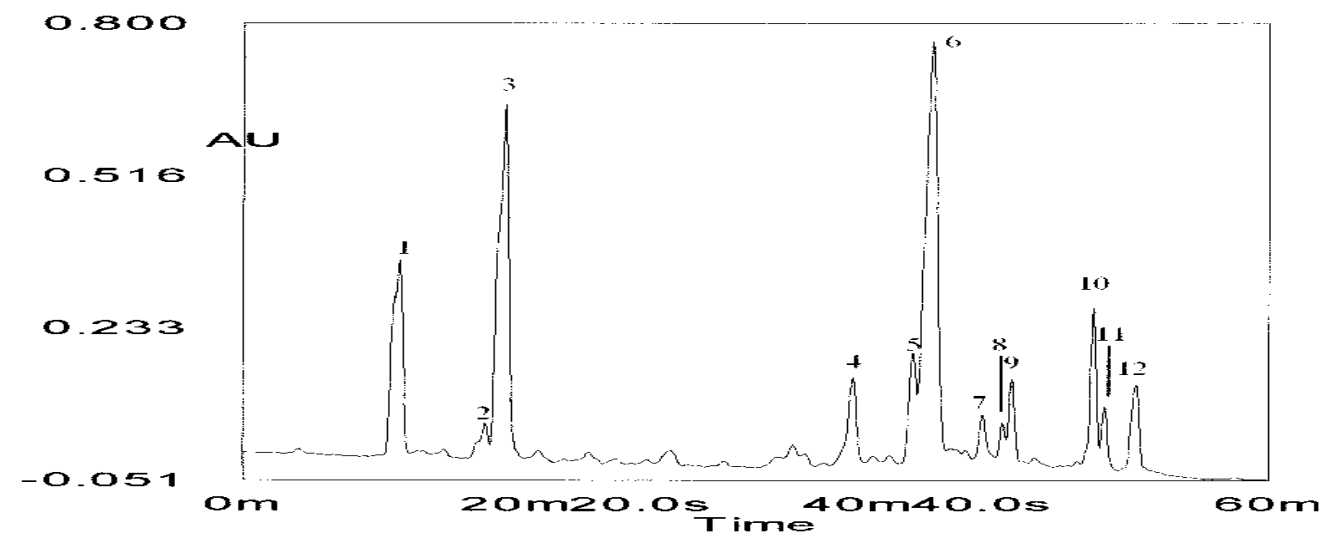

Figure 5. HPLC profile of a Cydonia oblonga Standards. Detection at $350 \mathrm{~nm}$. Peaks: (1) 3-Ocaffeoylquinic acid; (2) 4-O-caffeoylquinic acid; (3) 5-Ocaffeoylquinic acids; (4) 3,5dicaffeoylquinic acid; (5) Quercetin 3-galactoside; (6) Rutin; (7) Kaempferol glycoside; (8) Kaempferol 3-glucoside; (9) Kaempferol 3-rutinoside; (10) and (11) Quercetin glycosides acylated with $p$-coumaric acid, and (12) Kaempferol glycoside acylated with $p$-coumaric acid.

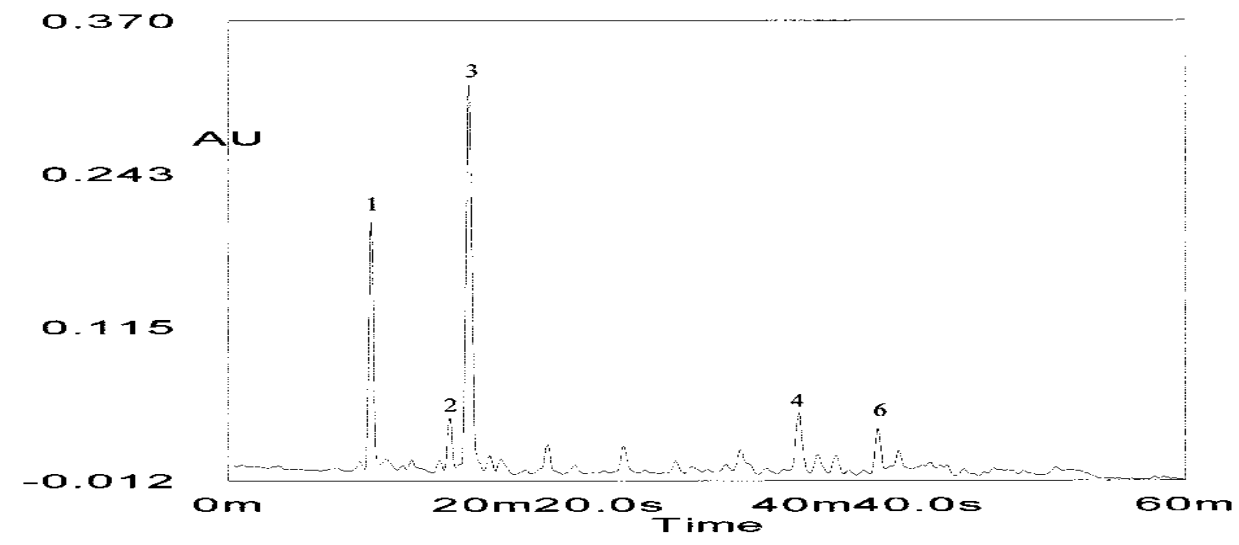

Figure 6. HPLC profile of Cydonia oblonga Extract. Detection at $350 \mathrm{~nm}$. Peaks: (1) 3-O-caffeoylquinic acid; (2) 4-O-caffeoylquinic acid; (3) 5-Ocaffeoylquinic acids; (4) 3, 5-dicaffeoylquinic acid, and (6) Rutin.

\subsection{Prunus avium (Cherry)}

Figure 7 depicts the chromatogram of the standards of Prunus avium.

A total of 11 phenolic compounds, hydroxycinnamic acid derivatives (3), anthocyanins (5), flavan-3-ols (2) and flavonol (1), were identified and quantified in sweet cherry cultivars. Three different hydroxycinnamic acid derivatives (neochlorogenic acid, p-coumaroylquinic acid and chlorogenic acid) were detected and quantified in the cherry (Fig. 8). Among the hydroxycinnamic acids, neochlorogenic acid showed the highest concentration of the total hydroxycinnamic acid content. Goncalves et al., (2004) reported that neochlorogenic acid was the major hydroxycinnamic acid, varying from 22 to $190 \mathrm{mg} / 100 \mathrm{~g}$ of $\mathrm{FW}$ and represented $19 \%$ to $71 \%$ of phenolics (Goncalves et al., 2004). Chlorogenic acid was the least abundant hydroxycinnamic acid. When we compare the total hydroxycinnamic acid derivative content of sweet cherries analysed with the previous reports, we see that our results were higher than the values obtained by Usenik et al., (2008) while lower than the values obtained by Goncalves et al., (2004). It was noted that cyanidin 3-rutinoside was the most dominant anthocyanin and it accounted for the largest proportion of the total anthocyanin contents. 


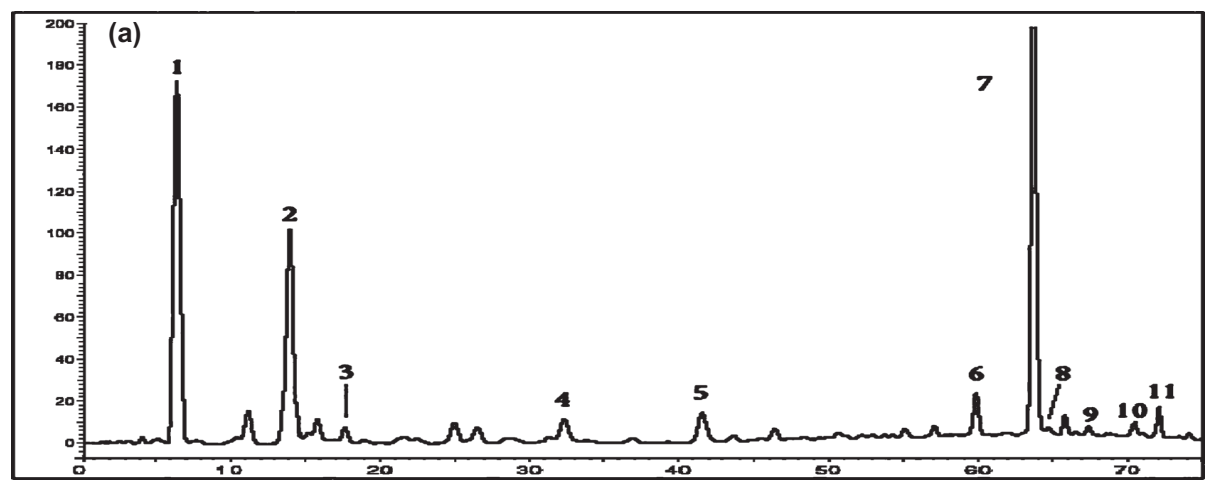

Figure 7. HPLC profile of Standards of Prunus avium. (1) Neochlorogenic acid; (2) pcoumaroylquinic acid; (3) Chlorogenic acid; (4) Cyanidin 3-glucoside; (5) Cyanidin 3rutinoside; (6) Peonidin 3-glucoside; (7) Pelargonidin 3-rutinoside; (8) Peonidin 3-rutinoside;

(9) Catechin; (10) Epicatechin; (11) Rutin

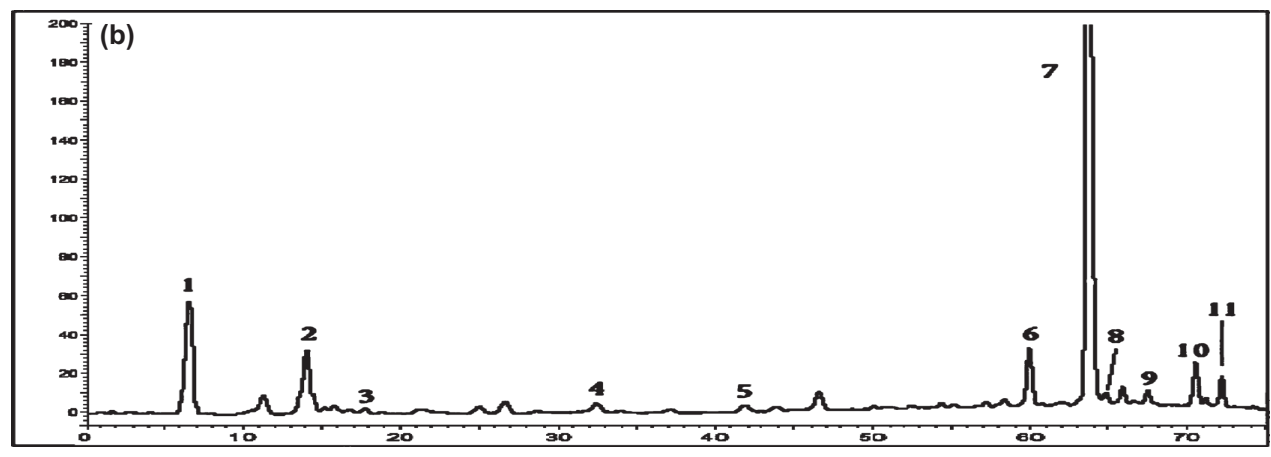

Figure 8. HPLC chromatograms of Prunus avium Extract recorded at $280 \mathrm{~nm}$.

\section{Conclusions}

The HPLC gradient mobile phase was developed to separate different types of phytochemicals in the selected indigenous fruits and vegetables. The choice of mobile phase and buffer (organic matter and $\mathrm{pH}$ ) composition plays an important role in the selectivity of separation. The final optimization can be done by changing the temperature, gradient slope and flow rate, and the type and concentration of the mobile phase modifier.

\section{References}

Bastos, C., Barros, L., Duenas M. (2015). Chemical characterisation and bioactive properties of Prunus avium L.: the widely studied fruits and the unexplored stems. Food Chemistry, 173, 1045- 1053.

Bystricka, J., Musilova, J., Lichtnerova, H., Lenkova, M., Kovarovic, J., Chalas, M. (2017). The content of total polyphenols, ascorbic acid and antioxidant activity in selected varieties of quince (Cydonia oblonga Mill.). Potravinarstvo Slovak Journal of Food Sciences, 11, 77-81.
Castejon, M. G., Visioli, F., Casado, A. R. (2012). Diverse biological activities of dandelion. Nutrition Reviews, 70 (9), 534547.

Chen, J. P., Tai, C. Y., Chen, B. H. (2004). Improved liquid chromatographic method for determination of carotenoids in Taiwanese mango (Mangifera indica L.). Journal of Chromatography $A$, 1054, 261-268

Chen, P., Tai, C. Y., Chen, B. H. (2004). Improved liquid chromatographic method for determination of carotenoids in Taiwanese mango (Mangifera indica L.). Journal of Chromatography $A$, 1054, 261-268.

Colle, D., Arantes, L. P., Gubert, P., Da Luz, S. C., Athayde, M. L., Teixeira, R. J. B., Soares, F. A. (2012). Antioxidant properties of Taraxacum officinale leaf extract are involved in the protective effect against hepatoxicity induced by acetaminophen in mice. Journal of Medicinal Food, 15(6), 549-556.

Crozier, A., Jaganath, Clifford, M. N. (2009). Dietary phenolics: chemistry, 
bioavailability and effects on health. Natural Product Reports, 26, 1001-1043.

Dugo, P., Herrero, M., Kumm, T., Giuffrida, D., Dugo, G., Mondello, L. (2008). Comprehensive normal-phase $\mathrm{x}$ reversed-phase liquid chromatography coupled to photodiode array and mass spectrometry detection for the analysis of free carotenoids and carotenoids esters from mandarin. Journal of Chromatography A, 1189, 196-206.

Fahimi, S., Mortazavi, S. A., Abdollahi, M., Hajimehdipoor, H. (2016). Formulation of a traditionally used polyherbal product for burn healing and HPTLC fingerprinting of its phenolic contents. Iranian Journal of Pharmaceutical Research, 15, 95-105.

Fattouch, S., Caboni, P., Coroneo,V., Tuberoso, C., Angioni, A., Dessi, S. (2007). Antimicrobial activity of Tunisian quince (Cydonia oblonga Miller) pulp and peel polyphenols extracts. Journal of Agricultural and Food Chemistry, 55, 963-969.

Goncalves, B., Landbo, A. K., Knudsen, D. (2004). Effect of ripeness and postharvest storage on the phenolic profiles of cherries (Prunus avium L.). Journal of Agricultural and Food Chemistry, 52, 523-530.

Guder, A., Korkmaz, H. (2012). Evaluation of in-vitro Antioxidant Properties of Hydroalcoholic Solution Extracts Urtica dioica L., Malva neglecta Wallr. and Their Mixture. Iranian Journal of Pharmaceutical Research, 11(3), 913-923.

Hamauzu, Y., Yasui, H., Inno, T., Kume, C., Omanyuda, M. (2005). Phenolic profile, antioxidant property, and anti-influenza viral activity of Chinese quince (Pseudocydonia sinensis Schneid.), quince (Cydonia oblonga Mill.), and apple (Malus domestica Mill.) fruits. Journal of Agricultural and Food Chemistry, 53, 928-934.

Inbaraj, B. S., Chien, J. T., Chen, B. H. (2006). Improved high performance liquid chromatographic method for determination of carotenoids in the microalga Chlorella pyrenoidosa. Journal of Chromatography A, 1102, 193-199.
Inbaraj, B. S., Lu, H., Hung, C. F., Wu, W. B., Lin, C. L. Chen, B. H. (2008). Determination of carotenoids and their esters in fruits of Lycium barbarum Linnaeus by HPLC-DAD-APCI-MS. Journal of Pharmaceutical and. Biomedical Analysis, 47, 812-818.

Joana, G., Rodrigo, R., Angelo, L., Sandra, R., Tiago, R., Eugenia, G., Ana, P. D. (2019). Assessment of the Bio accessibility and Bioavailability of the Phenolic Compounds of Prunus avium L. by in Vitro Digestion and Cell Model. ACS Omega, 4, 7605-7613.

Karak, P. (2019). Biological activities of flavonoids: An overview. International Journal of Research in Pharmaceutical Sciences, 10(4), 1567-74.

Kenny, O., Brunton, N. P., Walsh, D., Hewage, C. M., McLoughlin, P., Smyth, T. J. (2015). Characterisation of antimicrobial extracts from dandelion root (Taraxacum officinale) using LCSPENMR. Phytotherapy Research, 29(4), 526-532.

Liu, R. H. (2013). Dietary bioactive compounds and their health implications. Journal of Food Science, 78, 18-25

Melendez-Martinez, A. J., Britton, G., Vicario, I. M., Heredia, F. J. (2008). The complex carotenoid pattern of orange juices from concentrate. Food Chemistry, 109, 546-553.

Monteiro Egydio, A. P., Santa Catarina, C., Segal, F. E. I., Cursino, A., Dos, S. D.Y. (2013). Free amino acid composition of Annona (Annonaceae) fruit species of economic interest. Industrial Crops and Products, 45, 373-376.

Mousavi, S. N. M., Delazar, A., Nazemiyeh, H., Khodaie, I. (2015). Biological activity and phytochemical study of Scutellaria platystegia. Iranian Journal of Pharmaceutical Research, 14, 215-23.a

Nagahora, N., Ito, Y., Nagasawa, T. (2013). Dietary Chinese quince polyphenols suppress generation of $\alpha$-dicarbonyl compounds in diabetic KK-Ay mice. Journal of Agricultural and Food Chemistry, 61, 6629-6635.

Nesrin, H., Abdulselam, E., Elif, V.O., Hüseyin, A., Mehmet, B., Mustafa, A.Y., İsmail, Y.(2017). Chemical Profile of 
Malva neglecta and Malvella sherardiana by LCMS/MS, GC/MS and their anticholinesterase, antimicrobial and antioxidant properties with aflatoxincontents. Marmara Pharmaceutical Journal, 21(3), 471-484.

Serra, A. T., Duarte, R. O., Bronze, M. R., Duarte, C. M. M. (2011). Identification of bioactive response in traditional cherries from Portugal. Food Chemistry, 125, 318325.

Strek, M., Gorlach, S., Podsedek, A., Sosnowska, A. D., Koziolkiewicz, M., Hrabec, Z. (2007). Procyanidin oligomers from Japanese quince (Chaenomeles japonica) fruit inhibit activity of MMP-2 and MMP-9 metalloproteinases. Journal of Agricultural and Food Chemistry, 55, 6447-6452.

Toydemir, G., Boyacioglu, D., Capanoglu, E. (2013). (Investigating the transport dynamics of anthocyanins from unprocessed fruit and processed fruit juice from sour cherry (Prunus cerasus L.) across Intestinal epithelial cells. Journal of Agricultural and Food Chemistry, 61, 11434- 11441.

Tural, S., Koca, I. (2008). Physico-chemical and antioxidant properties of cornelian cherry fruits (Cornus mas L.) grown in Turkey. Scientia Horticulturae, 116, 362366.

Usenik, V., Fabcic, J., Stamper F. (2008). Sugars, organic acids, phenolic composition and antioxidant activity of sweet cherry (Prunus avium L.). Food Chemistry, 107, 185-192.

Vagelas, I., Sugar, I. R. (2020) Potential Use of Olive Oil Mill Waste Water to Control Plant and Post Harvest Diseases, Carpathian Journal of Food Science and Technology, 12, 140-144.

Wang, M., Jiang, N., Wang, Y. (2017). Characterization of phenolic compounds from early and late ripening sweet cherries and their antioxidant and antifungal activities. Journal of Agricultural and Food Chemistry, 5413-5420.

Wojdylo, A., Oszmianski, J., Bielicki, P (2013). Polyphenolic composition, antioxidant activity, and polyphenol oxidase (PPO) activity of quince (Cydonia oblonga Miller) varieties. Journal of Agricultural and Food Chemistry, 61, 2762-2772.

Zepka, L. Q., Mercadante, A. Z. (2009). Degradation compounds of carotenoids formed during heating of a simulated cashew apple juice. Food Chemistry, $117,28-34$.

\section{Conflicts of Interest:}

The authors declare that there are no conflicts of interest regarding the publication of this paper.

\section{Acknowledgments:}

The authors are highly thankful to the Department of Chemistry, KL DAV PG College Roorkee Uttrakhand India \& High End Instrumentation Lab, Public Health Laboratory Dalgate Srinagar J \& K India for providing facilities of the current work.

\section{Funding Statement:}

The research work did not received any funding from any source. This work was a part of a research work for awarding $\mathrm{PhD}$ degree. 